\title{
Über das Vorkommen von Milchsäure bei der Eklampsie.
}

Von

A. ten Doesschate.

(Aus dem physiologischen Laboratorium der Universität Utrecht.)

(Der Redaktion zugegangen am 1. Dezember 1907.)

In 1905 hat $\mathrm{Z}_{\text {weif }} \mathrm{e}^{1}$ ) Untersuchungen publiziert, aus welchen hervorgeht, daß bei der Eklampsie im Blut und Harn der Mutter sowie im Nabelstrangblut des Kindes Paramilchsäure in bedeutender Quantität enthalten sei, und er glaubt darin das die Krampfanfälle auslösende Gift gefunden zu haben.

Mein Zweck war es, diese Ergebnisse und die daraus gezogenen Schlußfolgerungen nachzuprüfen.

Zuerst wurde dazu die Literatur über das Vorkommen und die Entstehung von Milchsäure im Tierorganismus durchgesehen. Es gibt eine sehr große Zahl von Fällen, in denen Milchsäure gefunden sein soll; oft ist aber der Beweis nicht gebracht, daß die gefundene Substanz auch Milchsäure war. Sicher ist Paralactat beim Menschen vorgefunden im Blut bei der Leiche, bei Leukämie und im blutigen Exsudat bei Pleuritis carcinomatosa, in Blut, Harn und Cerebrospinalflüssigkeit bei Eklampsie, ${ }^{2}$ ) im Harn nach starker Muskelarbeit, bei der Phosphorvergiftung, bei der akuten Leberatrophie, bei Epilepsie,

1) Z w eifel, Zur Aufklärung der Eklampsie, Archiv für Gynäkologie, Bd. LXXII, S. 1, und Bd. LXXVI, S. 536. 1906, Nr. 7.

2) Zweifel, Das Gift der Eklampsie, Münch. Med. Wochenschr.,

Lockemann, Milchsäure in Blut, Urin und Cerebrospinalflüssigkeit bei Eklamptischen, Münch. Med. Wochenschr., 1906, Nr. 7.

Füth und Lockemann, Über den Nachweis von Fleischmilchsäure in der Cerebrospinalflüssigkeit Eklamptischer, Zentralbl. f. Gynäkol., 13. Jan. 1906. 
Hyperemesis gravidarum, und bei Lungenkranken im letzten Lebensstadium; des weiteren noch in Gehirn, Darminhalt und in Empyemeiter.

Die inaktive Milchsäure ist gefunden worden in Gehirn, Darminhalt und Urin. Schließlich ist Milchsäure, ohne Bestimmung, ob Para- oder Gärungsmilchsäure, im Urin gefunden bei der Epilepsie und in einem Fall von niedrigem Hämoglobingehalt des Blutes, sowie in verweichtem Knochen.

Die Frage, woraus die Milchsäure gebildet werde, ist nicht gelöst; daß sie aus Kohlehydraten entstehe, ist im Organismus nicht mit absoluter Sicherheit beobachtet worden, dagegen ist es bewiesen, daß Eiweiß die Muttersubstanz sein kann. Die vielen Gifte, welche, wenn sie in den Organismus einverleibt werden, die Entstehung von Milchsäure verursachen, geben in den meisten Fällen Veranlassung, entweder zu einer Destruktion von Eiweiß, oder zu Sauerstoffmangel, welch letzterer wieder das Zugrundegehen von Eiweiß hervorruft. Es lassen sich die verschiedenen Beobachtungen ungefähr so zusammenstellen. Bei mehreren Tieren findet man im Blut eine kleine Menge Milchsâure, welche gebildet wird bei der Muskelarbeit und wahrscheinlich in verschiedenen sonstigen Organen; die Säure entsteht aus Eiweiß, vielleicht auch aus Kohlehydraten, und geht nicht in den Harn über. Bei der weiteren Umbildung der Milchsäure spielt die Leber eine Rolle; gibt es kein funktionierendes Leberparenchym mehr, so steigt der Milchsâuregehalt des Blutes, und erscheint die Säure auch im Harn. Dasselbe findet auch statt bei angestrengter Muskelarbeit und bei erhöhter Eiweißspaltung, wie diese durch eine Reihe von Giften und durch Sauerstoffmangel hervorgerufen wird.

Zweifel nun fand in 17 Fällen von Eklampsie ohne Ausnahme Zinkparalactat im Urin, bisweilen nur eine Spur, meistens jedoch eine meßbare Quantität, bis zu 1\%o. Im Aderlaßblat von eklamptischen Mültern wurde von $0-1,1 \%$ gefunden. Da nun aber auch bei der Epilepsie und bei der Strychninvergiftung Milchsäure im Harne gefunden worden ist, könnie man das Vorkommen der Säure bei Eklamptischen auch den 
Konvulsionen und der Dyspnoe zuschreiben. Es wurde deshalb die Milchsäure unter anderen Verhältnissen gesucht, und zwar im Blut der Kinder von eklamptischen Müttern und bei der Graviditätsnephritis. Im Nabelstrangblut wurde fast immer Milchsäure gefunden, bis zu $0,8 \%$; auch das aus den Placenten stammende Blut lieferte bis $2 \%$ ioo Zinkparalactat. Bei der Graviditätsnephritis wurden im Harn immer Krystalle gefunden; jedoch war die Menge für die chemische Bestimmung zu gering. Dagegen lieferte Aderlaßblut in zwei untersuchten Fällen 0,076 und $0,25 \%$ Lactat.

Bei meiner Arbeit habe ich die von $\mathrm{Z} w$ eifel beschriebene Methode benützt, welche im wesentlichen mit derjenigen übereinstimmt, welche Araki ${ }^{1}$ ) angegeben hat. Die Placenten wurden nicht von den Nabelarterien aus durchspült, sondern zerhackt, mit Wasser gemischt und ausgepreßt.

Zur Identifikation der erhaltenen Substanz habe ich, wenn mehr als $100 \mathrm{mg}$ zu meiner Verfügung standen, mit einer ein zelnen Ausnahme immer die Bestimmung des $\mathrm{ZnO}$-Gehaltes ausgeführt, in den meisten Fällen auch die Bestimmung des Krystallwassergehalts. Oft aber standen mir nur kleine Quantitäten zu Gebote; es wurde dann zuerst die Krystallform beachtet, welche abwechselnd Nadeln und Prismen zeigte, gesondert und in Krystalldrusen. Sammelt man die von mehreren Fällen stammende Substanz, und führt man damit die Aschebestimmung aus, so hat zwar ein positives Resultat seinen Wert, ein negatives beweist jedoch nicht, daß nicht einige von den Teilen Zinkparalactat gewesen sind. Ich habe es deshalb bevorzugt, in diesen Fällen die von Hopkins ${ }^{2}$ ) angegebene Probe auszuführen: In einem Reagenzglas wird eine kleine Quantität der zu untersuchenden Substanz gemischt, mit $5 \mathrm{ccm}$ starker Schwefelsäure und einem Tropfen gesättigter Lösung von Kupfersulfat 1-2 Minuten gekocht, schnell abr gekühlt und 2-3 Tropfen einer Thiophenlösung (10-20 Tropfen

1) Über die chemischen Änderungen der Lebensprozesse infolge von Sauerstoffmangel, Diese Zeitschrift, Bd. XIX, 1894, S. 335.

2) Fletscher and Gowland Hopkins, Lactic acid in amphibian muscle, Journal of Physiology, Vol. XXXV, p. 247, 1907. 
Thiophen in $100 \mathrm{ccm}$ Alkohol) hinzugefügt; das Reagenzglas wird nun wieder in kochendes Wasser gebracht; ist Milchsäure anwesend, so entsteht eine kirschrote Verfärbung, welche beim Abkühlen nicht verschwindet. Die Reaktion wird nicht gegeben durch Kohlehydrate, Acetaldehyd und viele untersuchte physiologische Produkte: wohl dagegen durch Malonsäure und wahrscheinlich durch andere $\alpha$-Oxysäuren als die Milchsäure. .Von diesen werden in Hammarstens Lehrbuch der physiologischen Chemie 1907 nur die Uroleucinsäure und die Oxymandelsäure genannt; erstere ist nur bei der Alkaptonurie im Urin vorgefunden; mit dem Millonschen Reagens liefert sie ein gelbes Präzipitat, das beim Erwärmen sich rötet; die Oxymandelsäure ist einmal bei akuter gelber Leberatrophie im Harn gefunden worden von Schultzen und RieB $;^{1}$ ) sie soll beim Erhitzen mit $\mathrm{Ca}(\mathrm{OH})_{2}$ Phenol liefern. Mit der Substanz, welche ich aus dem Harn einer Eklamptischen erhalten hatte, habe ich diese Proben angestellt, beide mit negativem Resultat. Wenn also die Probe von Hopkins positiv ausfällt, darf sie wohl als beweisend für die Anwesenheit von Milchsäure gelten. Das Umgekehrte ist nicht der Fall; in zwei Fällen fiel die Probe negativ aus, in welchen die $\mathrm{ZnO}$-Bestimmung den Beweis erbracht, daß die untersuchte Substanz Milchsäure war; hier waren die Krystalle aus Urin erhalten, vielleicht wurde die Reaktion von Urinfarbstoffen gehindert.

In Fällen mit vielen Verunreinigungen, in welchen das Waschen mit absolutem Alkohol nicht genügte, mußten die Krystalle bisweilen mit Tierkohle gereinigt werden. Um die dabei verlorene Quantität kennen zu lernen, führte ich viermal diese Reinigung aus mit einer gewogenen Menge Zinkparalaktat; so fand ich eine Abnahme von resp. 2,5\%, 8,3\%, 2,66 $\%$ und $4 \%$. Bei der Berechnung der gefundenen Quantität Zinkparalaktat habe ich darum für den mit der Reinigung statthabenden Verlust $4 \%$ gerechnet. Um meine Zahlen mit den von $\mathrm{Z}$ weifel gegebenen vergleichen zu können, habe ich ebenso wie er die spezifische Schwere von Blut und Urin auf

1) Akute Phosphorvergiftung und akute Leberatrophie, CharitéAnnalen, Berlin, Bd. XV, 1869, S. 1. 
1000 gestellt und alles als lufttrockenes, krystallwasserhaltendes Zinkparalaktat berechnet.

Ich konnte 8 Fälle von Eklampsie untersuchen.

I. 5. Nov. 1906. VIIpara. 11 Anfälle. Gestorben.

Aus $330 \mathrm{ccm}$ Urin Krystalle: Rosette von Prismen; der gereinigte Teil hiervon wiegt $144 \mathrm{mg}$. Dies verliert bei $110^{\circ}$ : $18,8 \mathrm{mg}=13,05 \%$. Geglüht liefert es $41,9 \mathrm{mg} \mathrm{ZnO}=33,46 \%$. Für Zinkparalaktat berechnet sind diese Zahlen: 12,89\% und $33,43 \%$. Die gefundene Substanz ist also Zinkparalactat, und zwar $0,044 \%$ von dem Urin.

Die Placenta wiegt $215 \mathrm{~g}$. Gefunden: $251,3 \mathrm{mg}$ Substanz. Krystallwasser: $32,9 \mathrm{mg}=13,09 \%$. Zn0: 73,3 mg $=33,56 \%$. Also Zinkparalactat, und zwar 0,11\%.

In $45 \mathrm{ccm}$ mütterlichem Blut: Krystalle in Drusen: Bei $110^{\circ}$ getrocknet: $79,6 \mathrm{mg}$; Probe von Hopkins positiv. Also wahrscheinlich Paralactat; auf das lufttrockene Salz berechnet $0,2 \%$.

In $40 \mathrm{ccm}$ Nabelstrangblut des toten Kindes: bei $110^{\circ}$ getrocknet: 42,2 mg Krystalle. Hopkins positiv. Also lufttrockenes Salz: 0,121\%. Diese Krystalle werden vereinigt mit der Substanz, welche noch aus dem Urin übriggeblieben ist; zusammen: $132,3 \mathrm{mg}$. Krystallwasser: $15 \mathrm{mg}=11,34 \%$. Geglüht: $50,6 \mathrm{mg}=43,14 \%$. Der große Fehler in der $\mathrm{ZnO}$ Bestimmung wird hier vielleicht von dem aus dem Harn stammenden Teile verursacht.

II. 29. Nov. Vpara. 7 Anfälle. Genesung.

Urin untersucht vom 28. Nov., 30. Nov., 1. Dez., 3. Dez. und 5. Dez. Immer wurden Krystalle gefunden in Form von feinen Nadeln. Wiegen zusammen: $67,1 \mathrm{mg}$. Verlust bei $110^{\circ}$ : $5,1 \mathrm{mg}=7,6 \%$. Nach der Glühung: $48,8 \mathrm{mg}=78,7 \%$. Die Substanz war also kein Paralactat; zwar war die Hopkins sche Probe deutlich positiv; also wenigstens eine Spur Milchsäure.

III. 11. Jan. 1907. 5 Anfälle. Genesung.

In $45 \mathrm{ccm}$ Urin vom 11. Jan. Krystalle: Nadeln in Büscheln. In $675 \mathrm{ccm}$ Urin vom 15. Jan.: Krystalle, zusammen mit denjenigen vom 11. Jan. bei $110^{\circ}$ getrocknet: $209,1 \mathrm{mg}$, jedoch 
noch nicht rein, obgleich mit Tierkohle behandelt. Hopkins positiv.

Aus $26 \mathrm{ccm}$ Blut des Kindes typische Krystalle: lange verzweigte Nadeln. Hopkins +. $90 \mathrm{mg}$ verlieren bei $110^{\circ}$ : $7,6 \mathrm{mg}=8,44 \%$; enthalten $55,2 \mathrm{mg} \mathrm{ZnO}=66,99 \%$. Also eine Spur Paralactat.

IV. 12. Jan. Ipara. 4 Anfälle. Genesung.

Im mütterlichen Blut: bei $110^{\circ}$ getrocknete Krystalle: $62,2 \mathrm{mg}$.

Im Nabelstrangblut: $7,2 \mathrm{mg}$.

In $40 \mathrm{ccm}$ Urin bei $110^{\circ}: 128,2 \mathrm{mg}$. Diese drei Quantitäten gesammelt, umkrystallisiert und mit Tierkohle gereinigt liefern zusammen $94,4 \mathrm{mg}$ lufttrockenes Salz. Verlust bei $110^{\circ}$ : $7,8 \mathrm{mg}=8,26 \% . \quad \mathrm{ZnO}: 43,6 \mathrm{mg}=50,35 \%$. Dagegen ist Hopkins + .

Die Placenta wiegt $685 \mathrm{~g}$; hieraus $1108,1 \mathrm{mg}$ Krystalle.

Ein gereinigter Teil wiegt $806 \mathrm{mg}$; Verlust bei $110^{\circ}$ : $104,2 \mathrm{mg}=12,92 \% ; \mathrm{ZnO}: 238,8 \mathrm{mg}=34,02 \%$. Der Rest wiegt bei $110^{\circ}$ noch $263,2 \mathrm{mg}$ und gibt die Reaktion von Hopkins. Zusammen also $0,162 \%$ vom Placentargewicht.

$7,20 \mathrm{ccm}$ Urin vom 17. Jan. liefern Krystalle, von denen ein Teil 175,7 mg wiegt; Verlust bei $110^{\circ} 7,3 \mathrm{mg}=4,15 \%$; $\mathrm{Zn0}: 104,5 \mathrm{mg}=62,05 \%$. Der Rest beträgt $35,6 \mathrm{mg}$; Verlust 4,6 mg =12,92\%. $\mathrm{ZnO}: 20,8 \mathrm{mg}=67,09 \%$. Hopkins + .

V. 24. Jan. 3 Anfälle. Genesung.

In $300 \mathrm{ccm}$ Urin $111 \mathrm{mg}$ Krystalle, von denen $68 \mathrm{mg}$ bei $110^{\circ} 8,8 \mathrm{mg}$ verlieren $=12,89 \%$. Hopkins + .

In $100 \mathrm{ccm}$ mütterlichem Blut $63,8 \mathrm{mg}$ Krystalle; Verlust $8 \mathrm{mg}=12,54 \%$. Hopkins + .

In $10 \mathrm{ccm}$ Nabelstrangblut $71,5 \mathrm{mg}$; Verlust $9,1 \mathrm{mg}$ $=12,73 \%$. Hopkins + .

Diese drei Quantitäten zusammen nochmals gereinigt wiegen bei $110^{\circ}: 104,3 \mathrm{mg} ; \mathrm{ZnO}: 71,7 \mathrm{mg}=68,7 \%$.

Die Placenta wiegt $365 \mathrm{~g}$, liefert $308 \mathrm{mg}$; Verlust bei $110^{\circ}: 39 \mathrm{mg}=12,66 \%$. $\mathrm{ZnO}: 92,1 \mathrm{mg}=34,24 \%$. Der nicht ganz reine Rest wiegt bei $110^{\circ}: 90 \mathrm{mg}$. Zusammen $0,11 \%$ vom Placentargewicht. 


\section{16. Febr. Vpara. 17 Anfälle. Genesung.}

Urin vom 14., 15., 17., 19 und 22. Febr.; enthält immer Krystalle. Die ganze Menge Substanz zusammengefügt wird dreimal mit Tierkohle gereinigt; Hopkins +. Ein reiner Teil wiegt $186,4 \mathrm{mg}$. Verlust $8,2 \mathrm{mg}=4,4 \% . \quad \mathrm{ZnO}: 61,6 \mathrm{mg}$ $=34,57 \%$. Der Rest wiegt bei $110^{\circ} 300,9 \mathrm{mg}$. Hiervon gibt $130,3 \mathrm{mg}$ nach der Glühung: $67,1 \mathrm{mg}=51,49 \%$. Also war nur der erste Teil Lactat; auf $4428 \mathrm{ccm}$ Urin berechnet: $0,004 \%$.

VII. 1. Mai. Ipara. 5 Anfälle. Genesung.

$614 \mathrm{ccm}$ Urin liefert, mit Kohle gereinigt, bei $110^{\circ}: 54,4 \mathrm{mg}$ Krystalle. Hopkins + .

$10 \mathrm{ccm}$ mütterliches Blut bei $110^{\circ}: 62,6 \mathrm{mg}$. Hopkins -.

$22 \mathrm{ccm} \mathrm{Nabelstrangblut} \mathrm{bei} 110^{\circ}: 53,8 \mathrm{mg}$. Hopkins + .

$615 \mathrm{~g}$ Placenta liefern 540,1 mg Krystalle. Verlust 69,4 mg $=12,85 \%$. $\mathrm{ZnO}: 155,7 \mathrm{mg}=33,08 \%$. Als Rest noch bei $110^{\circ}$ : 188,5 mg. Zusammen 0,123\%.

VIII. 24. Mai I para. 3 Anfälle. Genesung.

Aus $126 \mathrm{ccm}$ Urin sternförmige Krystalle, in einer von der gewöhnlichen abweichenden Form. Einmal mit Kohle gereinigt; bei $110^{\circ}$ : 70,5 mg. Hopkins + .

$280 \mathrm{ccm}$ mütterliches Blut: Büscheln von breiten, spitz endenden Nadeln. $110^{\circ}: 82,6 \mathrm{mg}$. Hopkins + .

$90 \mathrm{ccm}$ Nabelstrangblut, $110^{\circ}: 23,3 \mathrm{mg}$. Hopkins -

Placenta: $540 \mathrm{~g}$, liefert die typischen Krystalle. Einmal mit Kohle gereinigt. Lufttrocken: $141,5 \mathrm{mg}$. Verlust: $17,5 \mathrm{mg}$ $=12,37 \%$. $\mathrm{ZnO}: 42,5 \mathrm{mg}=34,27 \%$. Als Rest noch: $739,7 \mathrm{mg}$. Verlust: $69,3 \mathrm{mg}=9,37 \%$. ZnO: $242,8 \mathrm{mg}=36,22 \%$. Als nochmals geglüht wurde, um konstantes Gewicht zu erzielen, zerbrach der Tiegel. Wahrscheinlich ist also der Gehalt an ZnO geringer und ist die untersuchte Substanz Paralactat.

Folgende Tabelle gibt die Quantitäten des gefundenen Zinkparalactats, auch für jene Fälle, in welchen die $\mathrm{ZnO}$ Bestimmung nicht stattfand, die Form der Krystalle und die Probe von Hopkins es jedoch wahrscheinlich machten, daß die gefundene Substanz Paralactat war. Oft, wenn der Gehalt 


\begin{tabular}{|c|c|c|c|c|c|}
\hline & & $\begin{array}{c}\text { Probe } \\
\text { von } \\
\text { Hopkins }\end{array}$ & $\begin{array}{c}\text { Krystall- } \\
\text { wasser } \\
\text { in } \%\end{array}$ & $\begin{array}{l}\mathrm{ZnO} \\
\text { in } \%\end{array}$ & $\begin{array}{c}\text { Zn-para- } \\
\text { lactat } \\
\text { in } \%\end{array}$ \\
\hline \multicolumn{2}{|r|}{$\begin{array}{c}\text { Für reines Zinkparalactat be- } \\
\text { rechnet. }\end{array}$} & + & 12,89 & $\mathbf{3 3}, \mathbf{4 3}$ & \\
\hline \multirow[t]{5}{*}{ I. } & Mütterliches Blut. . . . . . & + & & & 0,2 \\
\hline & Nabelstrangblut . & + & & & 0,121 \\
\hline & Urin $\cdot . \cdot . \cdot \cdot \cdot \cdot \cdot \cdot$ & + & 13,05 & 33,46 & 0,044 \\
\hline & Rest des Urins + Nabelstrangblut & & 11,34 & 43,14 & \\
\hline & Placenta......... & & 13,09 & 33,56 & 0,11 \\
\hline II. & Urin . . . . . . . . & + & 7,6 & 78,7 & Spur \\
\hline \multirow[t]{2}{*}{ III. } & Nabelstrangblut . . . . . . & + & 8,44 & 66,99 & , \\
\hline & Urin . . . . . . . . & + & & & 0,035 \\
\hline \multirow{4}{*}{\multicolumn{2}{|c|}{$\begin{array}{l}\text { IV. Mütterliches Blut. . . . . . } \\
\text { Nabelstrangblut } . . \\
\text { Urin ante partum } . . . \\
\text { Die } 3 \text { vorhergehenden zusammen }\end{array}$}} & & & & Spur \\
\hline & & & & & $>$ \\
\hline & & & & & $\gg$ \\
\hline & & + & & & » \\
\hline \multirow{2}{*}{\multicolumn{2}{|c|}{$\begin{array}{l}\text { Urin post partum } . . . \\
\text { Rest des Urins post partum. . }\end{array}$}} & + & 4,15 & 62,05 & 》 \\
\hline & & & 12,92 & 67,09 & \\
\hline & Placenta . . . . . . . . & + & 12,92 & 34,02 & 0,162 \\
\hline & Mütterliches Blut. . . . . . & + & 12,54 & & Spur \\
\hline & Nabelstrangblut . . . . . . & + & 12,73 & & 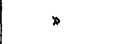 \\
\hline & Urin . . . . . . . . . & + & 12,89 & & $\triangleright$ \\
\hline & Die 3 vorhergehenden zusammen & + & & 68,7 & > \\
\hline & Placenta . . . . . . . & & 12,66 & 34,24 & 0,11 \\
\hline \multirow[t]{2}{*}{ VI. } & Urin $\cdot . \cdot \cdot \cdot \cdot \cdot \cdot \cdot$ & + & 4,4 & 34,57 & 0,004 \\
\hline & Rest des Urins . . . . . . . & & & 51,49 & \\
\hline \multirow[t]{4}{*}{ VII. } & Mütterliches Blut & - & & & 0 \\
\hline & Nabelstrangblut . . . . . . & + & & & 0,281 \\
\hline & Urin....... & + & & & 0,011 \\
\hline & Placenta . . . . . . . . . & + & 12,85 & 33,08 & 0,123 \\
\hline \multirow[t]{5}{*}{ VIII. } & Mütterliches Blut. . . . . . & + & & & 0,034 \\
\hline & Nabelstrangblut . . . . . . & - & & & 0 \\
\hline & Urin . . . . . . . . & + & & & 0,067 ? \\
\hline & Placenta . . . . . . . & + & 12,37 & 34,27 & 0164 \\
\hline & Rest der Placenta . . . . . & & 9,37 & 36,22 & \\
\hline
\end{tabular}


an $\mathrm{ZnO}$ nicht mit dem für Zinkparalactat berechneten übereinstimmte, zeigte doch die Farbenreaktion, daß wenigstens eine Spur Milchsäure anwesend war.

In den Fällen, in denen der Gehalt an $\mathrm{ZnO}$ nicht mit dem für Zinklactat berechneten übereinstimmte, war er immer höher, und zwar meistens von 50-70\%. In einigen von diesen Fällen fand beim Hinzufügen der Salpetersäure ein Aufbrausen statt; es war also wahrscheinlich $\mathrm{ZnCO}_{3}$ entstanden, welches $64,8 \% \mathrm{ZnO}$ enthält. Das $\mathrm{ZnCO}_{3}$ kann erst später entstanden sein, weil ursprünglich von einer klaren Lösung der Substanz in Wasser ausgegangen wird. Wahrscheinlich entsteht es aus organischen Substanzen, welche bei $110^{\circ}$ oxydiert werden; öfters mußten die Krystalle lange Zeit auf $110^{\circ}$ erhitzt werden, bevor das Gewicht konstant war; bisweilen währte dies mehr als 20 Stunden. Ich überzeugte mich, daß reines Zinkparalactat, auch wenn es während vieler Tage bis $110^{\circ}$ erhitzt wird, nicht in $\mathrm{ZnCO}_{3}$ übergeht.

Es ist möglich, daß in den Fällen, in welchen ein Gehalt an $\mathrm{ZnO}$ von mehr als $33 \%$ gefunden wurde, die Substanz aus einem Gemisch von Zinkparalactat mit etwas anderem bestanden habe.

In einem Falle (VI) wurde 4,4\% Krystallwasser gefunden. Weil aber der Wassergehalt des Zinkparalactats nicht ganz konstant ist, glaubte ich hier doch, auf die $\mathrm{ZnO}$-Bestimmung gestützt, annehmen zu dürfen, daß die untersuchte Substanz Zinkparalactat war.

In den von Eklamptischen stammenden Flüssigkeiten wurde also nur zweimal ke ine Milchsäure gefunden. Die Prozentzahlen stimmen ungefähr mit den von $\mathrm{Zw}$ eifel angegebenen überein.

In einigen Fällen hatten die Patienten Morphin bekommen; weil bei der Vergiftung von Tieren mit Morphin im Harn Milchsäure gefunden worden ist, untersuchte ich den Urin eines Morphinisten, welcher täglich $700 \mathrm{mg}$ subkutan bekam. Aus $676 \mathrm{ccm}$ erhielt ich $72,3 \mathrm{mg}$ Krystalle; Verlust bei $110^{\circ}$ : $3,8 \mathrm{mg}=5,26 \%$. Geglüht: $46,2 \mathrm{mg}=67,45 \%$. Es erübrigte ein Rest von $23,8 \mathrm{mg}$ (bei 110) ${ }^{\circ}$. Im Urin war also nur eine 
Spur Milchsäure, weil die Reaktion von Hopkins positiv war. Wir sind also zu dem Schluß berechtigt, daß die kleinen Quantitäten Morphin, welche die Patienten bekamen, keinen Einfluß auf die Werte des Lactats geübt haben.

Bei der Eklampsie wird also in Blut, Harn, Placenta und Nabelstrangblut fast immer Milchsäure gefunden. Es frägt sich nun, inwieweit das abnormal sei.

Im gewöhnlichen Harn konnten Liebig, Heuss, Schütz keine Milchsäure finden, im Einklang damit konnte ich aus 11 Urin keine Krystalle darstellen; auch zeigte 11 Urin, bis $10 \mathrm{ccm}$ eingeengt und mit Kohle gereinigt, die Reaktion von Hopkins nicht. - Dagegen fand ich die typischen Krystalle in $1350 \mathrm{ccm}$ Urin eines Patienten, welcher schwere Dyspnoe hatte. Außer einigen gefärbten Krystallen aus dem Waschalkohol wogen diese $49,2 \mathrm{mg}$. Verlust bei $110^{\circ}: 4,2 \mathrm{mg}=8,54 \%$. $\mathrm{ZnO}$ : $17,7 \mathrm{mg}=39,33 \%$. Hopkins + . Dies war also wahrscheinlich unreines Paralactat, welches $0,004 \%$ des Urins ausmachen würde.

Um einen Einblick in die Genauigkeit der Methode zu gewinnen, löste ich Zinkparalactat in normalem Harn. Sch ütz ${ }^{1}$ ) fand mit zwei verschiedenen Methoden resp. $35 \%$ und $56 \%$ zurück. . Stadelmann ${ }^{2}$ ) 14,2\%, $\mathrm{Zw}_{\text {wifel }}{ }^{3}$ ) von $500 \mathrm{mg}$ sehr wenig. - In 11 Urin löste ich $300 \mathrm{mg}$ Zinkparalactat; die Krystalle, welche ich erhielt, mußten öfters mit Kohle gereinigt werden, und wogen bei $110^{\circ} 78,2 \mathrm{mg}$; dies war jedoch noch kein reines Lactat; beim Glühen lieferte es $42,1 \mathrm{mg}=53,84 \%$. Auch aus dem Waschalkohol wurden noch Krystalle erhalten. In einem anderen Versuche, wo weniger Farbstoffe zugegen waren, wurde mehr erhalten. In 11 Harn wurden $300 \mathrm{mg}$ Paralactat (bei $110^{\circ}$ ) gelöst, welches aus Pferdefleisch bereitet war und $32,6 \% \mathrm{ZnO}$ enthielt. Bei der Bearbeitung ward etwas verloren. Die gefundenen Krystalle wurden einmal mit Kohle 1894, S. 482.

1) Milchsäure in patholog. Harnen, Diese Zeitschrift, Bd. XIX,

2) Ammoniakausscheidung beim Diabetes, Archiv f. exper. Pathol. u. Pharm., 1883, Bd. XIX, S. 419.

$\left.{ }^{3}\right)$ Arch. f. Gynäkol., Bd. LXXVI, S. 554. 
gereinigt; ein Teil wog bei $110 \%: 46,4 \mathrm{mg}$, ein anderer Teil $44,1 \mathrm{mg}$; dieser lieferte $15,6 \mathrm{mg} \mathrm{ZnO}=35,27 \%$. Wiewohl in diesem Fall die Hopkinssche Probe negativ ausfiel, zeigte doch Krystallform und ZnO-Bestimmung, daß die Substanz Paralactat sein mußte, obgleich noch nicht ganz rein. Im ganzen wurden also $90,5 \mathrm{mg}$ zurückbekommen, das heißt, wenn für den Verlust beim Reinigen mit Kohle $4 \%$ gerechnet wird, $94,2 \mathrm{mg}=31,4 \%$ von dem in dem Harn gelösten.

Normales Menschenblut enthält keine Milchsäure nach Enderlin ${ }^{1}$ ) und Salomon ${ }^{2}$ ). Auch $Z_{w}$ eifel fand es nicht im Aderlaßblut von Schwangeren und Gebärenden. Berlinerblau ${ }^{3}$ ) stellte aus $200 \mathrm{ccm}$ direkt in Alkohol aufgenommenem venösen Blut 24,5 mg Substanz dar, von welcher aber keine Bestimmung angegeben wird; auch wird nicht gemeldet, ob das Blut von einem gesunden Menschen stammte.

Ich untersuchte $100 \mathrm{ccm}$ Aderlaßblut von einem normalen Menschen, welcher in 16 Stunden keine Nahrung zu sich genommen hatte; $50 \mathrm{ccm}$ hiervon wurden mit Alkohol extrahiert; in der zweiten Hälfte wurde Zinkparalactat gelöst, welches bei $110^{\circ} 190,6 \mathrm{mg}$ wog und $32,6 \% \mathrm{ZnO}$ enthielt. Es wurde wieder erhalten: 89,5 mg; Verlust bei $110^{\circ}: 10 \mathrm{mg}=11,17 \%$; nach der Glühung: $26,4 \mathrm{mg}=33,21 \%$. Ein Rest war noch übrig, welcher bei $110^{\circ} 9,8 \mathrm{mg}$ wog. Zusammen wurden also $89,3 \mathrm{mg}$ wieder gefunden $=46,86 \%$.

Aus den $50 \mathrm{ccm}$ normalen Blutes erhielt ich Krystalle in zwei Formen, und zwar Drusen von Nadeln und von breiteren, kegelförmigen Krystallen. Nach Umkrystallisierung wurde ein rein weißer Teil erhalten: lufttrocken $20 \mathrm{mg}$; Verlust bei $110^{\circ}$ : $2,1 \mathrm{mg}=10,5 \% ; \mathrm{ZnO}: 15,4 \mathrm{mg}=86,03 \%$. Es war also kein Lactat; die Probe von Hopkins war aber unzweifelhaft positiv. Ein Rest wog bei $110^{\circ} 35,9 \mathrm{mg}$; Hopkins schwach. Das Blut enthielt also nur eine Spur Milchsäure.

") Über die milchsauren Salze im Blute, Li ebigs Annalen, Bd. XLVI, S. $164,1843$.

2) Verbreitung und Entstehung von Hypoxanthin und Milchsäure, Diese Zeitschrift, Bd. II, 1878, S. 65.

s) Milchsäure im Blute, Archiv f. exper. Path. u. Pharm., Bd. XXIII, 1887, S. 333 . 
Auch wurden zur Kontrolle Placentae untersucht. Weil es möglich wäre, daß bei lange dauernden Wehen durch Störungen im Placentar- und im kindlichen Kreislauf Milchsäure entstehe, untersuchte ich Placentae, welche von Geburten von verschiedener Dauer stammten.

Aus der Placenta von einer sehr kurz dauernden Geburt, welche zusammen mit dem Nabelstrangblut $470 \mathrm{~g}$ wiegt, werden Krystalle erhalten; nach Reinigung, bei $110^{\circ}: 24,3 \mathrm{mg}$. Hopkins + .

Placenta: $445 \mathrm{~g}$. Partus von 7 Stunden. $42,6 \mathrm{mg}$ Krystalle. Hopkins + .

Aus $36 \mathrm{ccm}$ Nabelstrangblut die typischen Krystalle von Zinkparalactat. Ein Teil wiegt $27,1 \mathrm{mg}$; Verlust $59,3 \mathrm{mg}$ $=21,88 \% ; \mathrm{ZnO}: 99 \mathrm{mg}=46,76 \%$. Rest wiegt $127,7 \mathrm{mg}$. Verlust $21,5 \mathrm{mg}=16,84 \% ; \mathrm{ZnO}: 51,9 \mathrm{mg}=48,87 \%$. Hopkins + .

Partus von 8 Stunden. Placenta $438 \mathrm{~g}$. Krystalle wiegen bei $110^{\circ} 34 \mathrm{mg}$. $\mathrm{ZnO} 22,8 \mathrm{mg}=67,06 \%$. Hopkins + .

Partus von 3 Tagen. Placenta $372 \mathrm{~g}$; das Nabelstrangblut wurde hinzugefügt. Krystalle: $91 \mathrm{mg}$. Verlust $6,5 \mathrm{mg}=$ $7,14 \%$. $\mathrm{ZnO} 44 \mathrm{mg}=52,07 \%$. Hopkins + .

$520 \mathrm{ccm}$ Urin von diesem Falle lieferten $42,8 \mathrm{mg} \mathrm{Kry-}$ stalle. Verlust $1,4 \mathrm{mg}=3,27 \%$. $\mathrm{ZnO} 27,3 \mathrm{mg}=65,94 \%$.

In den zwei letzten Fällen war also die gefundene Substanz kein Lactat. Wenn wir annehmen dürfen, daß die aus den ersten zwei Placentae erhallene Substanz Lactat war, so würde dies 0,006 und $0,012 \%$ betragen, also viel weniger als in den Placenten von Eklamptischen.

Bei einem normalen Partus (von 5 Stunden) wurde gefunden: In $83 \mathrm{ccm}$ Blut der Mutter Krystalle wie von Paralactat, und zwar $57,5 \mathrm{mg}$. Verlust $15,9 \mathrm{mg}=27,65 \%$. Hopkins + .

In $42 \mathrm{ccm} \mathrm{Nabelstrangblut} 45,2 \mathrm{mg}$. Verlust $3,2 \mathrm{mg}$ $=7,08 \%$. Hopkins + .

In $660 \mathrm{ccm}$ Urin bei $110^{\circ} 50,6 \mathrm{mg}$ Hopkins -

Im mütterlichen und kindlichen Blut war also wenigstens eine Spur Milchsäure enthalten. 
Ein anderer Partus wurde nach 90 Stunden mit der Zange beendet. Die einzige Abnormität ante partum war die ziemlich starke Obstipation der Frau.

In $400 \mathrm{ccm}$ mütterlichem Blut wurden $94,3 \mathrm{mg} \mathrm{Kry-}$ stalle gefunden; $\mathrm{H}_{2} \mathrm{O} 12,5 \mathrm{mg}=13,26 \%$. $\mathrm{ZnO} 28 \mathrm{mg}=$ $34,11 \%$. Dies war also Paralactat. Als Rest noch bei $110^{\circ}$ $11,8 \mathrm{mg}$. Zusamman auf lufttrockenes Salz berechnet 107,8 mg $=0,029^{\circ} \%$.

In $38 \mathrm{ccm}$ Nabelstrangblut keine Krystalle.

In $2830 \mathrm{ccm}$ Urin nach Reinigen mit Kohle bei $110^{\circ}$ 45,2 mg Krystalle. Hopkins +. Dies würde 0,002\% des Urins betragen.

Aus den Kontrollversuchen geht hervor, daß normaler Harn keine Milchsäure enthält, normales Blut eine Spur; daß von hinzugefügtem Paralactat aus dem Harn bis zu $31,4 \%$ wieder erhalten wurde, aus dem Blut 46,86\%. Weiter, daß bei normalen Geburten die Placenten eine Spur, oder jedenfalls sehr wenig Milchsäure enthalten, das Nabelstrangblut eine Spur oder nichts, der Harn nichts, das mütterliche Blut eine Spur; in letzterem wurde nur einmal eine ziemlich große Quantität Lactat gefunden, und zwar nach einem sehr lange dauernden Partus; in dem Falle war auch im Urin Milchsäure, im Nabelstrangblut dagegen nichts.

Die Quantität der Milchsäure, welche bisweilen bei der Eklampsie gefunden wird, ist also viel größer als unter normalen Umständen. Daß diese Milchsäure die Ursache der Krämpfe sei, versuchte $\mathrm{Zw}$ eifel aus der Tatsache abzuleiten, daß sie auch in jenen Fällen angetroffen wird, in welchen von Krämpfen keine Rede war, im Blut der Kinder von Eklamptischen, und bei Frauen mit Schwangerschaftsnephritis, bei welchen also Eklampsie ausbrechen kann. Das selbständige Vorkommen der Milchsäure im kindlichen Organismus, unabhängig von dem vielleicht durch die Krämpfe im mütterlichen Blut geformten Paralactats, ist nur in jenen Fällen anzunehmen; in welchen im kindlichen Blut ein höherer Gehalt als im mütterlichen gefunden wird.

Dies wurde von $\mathrm{Z}$ weifel dreimal, von mir einmal be- 
obachtet. Es scheint mir, daß der Schluß, es komme bisweilen im kindlichen Blut mehr Milchsäure vor als im mütterlichen, durch diese Wahrnehmungen nicht genügend gerechtfertigt sei. Erstens ist in keinem dieser Fälle die Bestimmung des Zn0Gehaltes der gefundenen Substanz einzeln ausgeführt. Zweitens sind die Quantitäten sehr gering, und sind also kleine Unterschiede von großem Einfluß auf die Zahl des Prozentsatzes. Diese kleinen Unterschiede werden leicht durch Verunreinigungen verursacht, welche zu Umkrystallisierung und Reinigung Anlaß geben, und weiter durch die Methode selber, welche quantitativ sehr mangelhaft ist, und mit welcher aus Blut weniger als $50 \%$ vom zugefügten Paralactat wiedergefunden wird.

In drei Fällen von Nephritis während der Gravidität wurde mit Sicherheit nicht mehr als eine Spur Milchsäure im Urin gefunden; auch $Z_{w}$ eifel erhielt zwar Krystalle, jedoch zu wenig für die Analyse. Im mütterlichen Blut fand er 0,0076 und $0,0252 \%$; im mütterlichen und kindlichen Blut fand ich nur eine Spur.

In den Placenten fand ich in einem Falle sehr viel: 0,119\%, also ebensoviel wie in den Placenten Eklamptischer. Dennoch bekam diese Frau keine Eklampsie. Hierbei war es bemerkenswert, daß 3 Tage nach der Geburt, bei welcher Zwillinge geboren wurden, das eine Kind starb unter Konvulsionen, während sein Urin eiweißhaltig war. Das zweite Kind blieb gesund.

Im Urin einer an Nephritis chronica erkrankten Frau, welche vor $2^{1 / 2}$ Monaten eine Eklampsie durchgemacht hatte, wurde noch eine Spur Milchsäure nachgewiesen.

Bei der Schwangerschaftsnephritis wird also in Blut, Harn und in der Placenta Milchsäure gefunden, und diese Säure würde also die Ursache der Krämpfe sein können.

Das Entgegengesetzte kann nur bewiesen werden, wenn es Fälle gibt, in welchen bei ebenso hohem Gehalt an Milchsäure keine Eklampsie auftritt. Dies ist nun in der Tat beobachtet. Im Urin der Eklamptischen wurde nur eine Spur gefunden, wie das unter vielen sonstigen Umständen vorkommt. Im mütterlichen Blut ward als höchster Gehalt von $\mathrm{Z}$ weifel $0,025 \%$ gefunden; bei einem normalen Partus fand ich 0,029\%. 
Auch in dem Fall; wo die Placenta $0,119 \%$ Paralactat enthielt, bekam die Frau keine Eklampsie; zwar starb das eine der Kinder unter Konvulsionen.

Die bei der Graviditätsnephritis vorkommende Milchsäure kann also das Entstehen der Krämpfe nicht erklären. Was nun die größere Quantität des Lactats anbelangt, welche im Harn gefunden wird, nachdem die Eklampsie ausgebrochen ist, so kann diese ganz durch die Krämpfe verursacht sein. Araki ${ }^{1}$ ) fand im Harn nach epileptischen Anfällen $0,439 \%$, während der höchste Gehalt bei der Eklampsie $0,1 \%$ beträgt. In ouye und $\mathrm{Saiki}^{2}$ ) fanden selbst nach einem epileptischen Anfalle bis zu 1\% Paralactat, in der Zeit zwischen den Anfällen nichts.

Der größte Gehalt an Zinkparalactat, welcher mit Sicherheit im mütterlichen Blut bei der Eklampsie beobachtet ist, beträgt $0,114 \%$. Obgleich nun in der Literatur außer in der Arbeit von $\mathrm{Zweifel} \mathrm{keine} \mathrm{Angaben} \mathrm{gefunden} \mathrm{werden} \mathrm{über} \mathrm{den}$ Milchsäuregehalt des Blutes von lebenden Menschen unter abnormen Umständen, so ist es doch sehr wahrscheinlich, daß auch da bisweilen viel Milchsäure im Blut ist, und zwar auf Grund der großen Quantitäten Lactat, welche im Urin gefunden worden sind; der Gehalt des Blutes ist in den meisten Fällen höher als der des Harns und auch bei Tieren wird normaliter im Urin keine, im Blut wohl Milchsäure gefunden. Bei Menschen kann nun der Urin sehr viel Milchsäure enthalten, ohne dab Krämpfe auftreten: bei Lungenkranken in Agone wurde 0,38\% gefunden, ${ }^{8}$ ) in einem Fall von Hyperemesis gravidarum 0,136\% 0.4 ) Diese Ziffern sind also höher als die höchste für das Blut bei der Eklampsie gefundene.

Die Quantität Milchsäure, welche bei der Eklampsie vorkommt, ist also wahrscheinlich nicht genügend zur Erklärung

1) Über die Bildung von Milchsäure und Glykose im Organismus bei Sauerstoffmangel, Diese Zeitschrift, Bd. XV, 1891, S. 335.

*) Über das Auftreten abnormer Bestandteile im Harn nach epileptischen Anfällen, Diese Zeitschrift, Bd. XXXVII, 1902, S. 203.

3) Irisawa, Milchsäure in Blut und Harn, Diese Zeitschrift, 1893, Bd. XVII, S. 340.

4) Underhill, A note on the presence of lactic acid in the urine of pernicious vomiting of pregnancy, The Journ. of biol. chem., Vol. II, p. 485, 1907.

Hoppe-Seyler's Zeitschrift f. physiol. Chemie. LIV. 
des Auftretens der Krämpfe; dagegen sind die Krämpfe imstande, den gefundenen Gehalt an Milchsäure zu verursachen:

Außer der Eklampsie und der Hypenemesis: hat man auch die Chorea gravidarum als eine Selwwangenschaftsintoxikation aufgefaßt. In einem: Fall von: Chorea; den ich untersuchent konnite, wurde gefunden: im mütterlichen und kindlichen Blut wenigstens eime Spur Milchsäure; in 11 Urin 0;0153\% \% Paralactat.

Der Schluß. zu: welchem ich gelangt bin, daß bei der Ektampsie die Käzmpfe nicht durch Anhäafung von Milchsäure verursacht werden; søndern umgekehrt selber za einer solchen Anltäufung Anlaß geben, läßt die Möglichkeit offen, daß die: Zunahme' der Quantität Milchsäłre im Blut Eklamptíscher außer von den Krämpfen auch nөch von anderen Emständen abhänge. Kommt doch auch bei der Graviditätsnephritis mehr Mlehsäure im Blut vor als in der Norm. Auch muß der Ändewungen gedacht werden; welche bei der Eklampsie in der Leber gefunden sind: Es liegt nahe; damit die Abnahme der Harnstoffund Sulfatausseheidung in Zusammenhang zu bringen. Ist nun Hei der Elklampsie die Leberfunktion wesentlich gestört, so: danfi man infolge davon eine Zunahme des Milchsäuregehaltb des: Blutes erwarten: Jedenfalls ist das Vorkommen einer exhöhten Quantität Milchsäure im Blut bei der Eklampsie ein Syreptom; dessen Unsprung, wenigstens hauptsächlich, klar ist; das. abolber eingtweilen nicht zur Erklärung der anderen Erscheinungen dieser Krankheit benütat werden kann. 